Revista Arbitrada Interdisciplinaria KOINONIA

Año V. Vol V. №9. Enero - Junio 2020

Hecho el depósito de Ley: FA2016000010

ISSN: 2542-3088

FUNDACIÓN KOINONIA (F.K). Santa Ana de Coro. Venezuela.

Hilter Farley Figueroa-Saavedra; Arisdorgan Diéguez-Almaguer; Aurora Azucena Ariza-Velasco

http://dx.doi.org/10.35381/r.k.v5i9.569

\title{
Propuesta de un Parque Ecológico para el fomento del Turismo
}

\section{Proposal of an Ecological Park for the Tourism promotion}

\author{
Hilter Farley Figueroa-Saavedra \\ hfigueroasaavedra@gmail.com \\ Escuela Superior Politécnica de Chimborazo, Extensión Norte Amazónica \\ Ecuador \\ https://orcid.org/0000-0002-7395-8944 \\ Arisdorgan Diéguez-Almaguer \\ arisdorgandieguez@gmail.com \\ Escuela Superior Politécnica de Chimborazo, Extensión Norte Amazónica \\ Ecuador \\ https://orcid.org/0000-0001-8361-7599 \\ Aurora Azucena Ariza-Velasco \\ aurora.ariza.velasco@gmail.com \\ Escuela Superior Politécnica de Chimborazo, Extensión Norte Amazónica \\ Ecuador \\ https://orcid.org/0000-0001-7024-0876
}

Recibido: 27 de octubre de 2019

Revisado: 10 de noviembre de 2019

Aprobado: 15 de diciembre de 2019

Publicado: 20 de enero de 2020

\section{RESUMEN}

La finalidad de la presente investigación fue, elaborar una propuesta para la creación de un Parque Ecológico adscrito al Consejo Provincial de Orellana para favorecer al turismo, La investigación lleva procesos que permiten gestionar los espacios naturales a través de planes que contienen medidas para la administración del espacio protegido, áreas de recreación y manejo. La finalidad de estas actuaciones es cumplir con los objetivos de conservación, expresado en la norma legal que recoge la declaración del espacio protegido. Para la aplicación del proyecto, cuenta con las políticas ambientales, contables, financieras que lleven a fomentar el turismo y mejorar la calidad de vida de la población a través de ella.

Descriptores: Ecología humana; comunidad; conservación ambiental; ecoturismo. (Palabras tomadas del Tesauro UNESCO). 


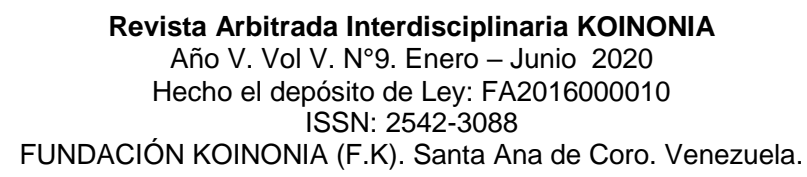

Hilter Farley Figueroa-Saavedra; Arisdorgan Diéguez-Almaguer; Aurora Azucena Ariza-Velasco

\begin{abstract}
The purpose of this research was to develop a proposal for the creation of an ecological park under the Orellana Provincial Council to promote tourism. The investigation considers processes that manage natural areas through plans containing measures for the administration of protected space, recreation areas and management. The purpose of these actions is to meet conservation objectives, expressed in the legal standards that includes the declaration of the protected area. For the implementation of the project, it counts on environmental, accounting and financial policies leading to promote tourism and improve the quality of people's life through this.
\end{abstract}

Descriptors: Human ecology; community; environmental conservation; ecotourism. (Words taken from the UNESCO Thesaurus).

\title{
INTRODUCCIÓN
}

En todo el planeta, las actividades de recreación y turismo en parques, áreas silvestres y áreas protegidas continúan mostrando tendencias de participación creciente (Monz; D'Antonio; Lawson; Barber and Newman, 2016). En la mayoría de los países en desarrollo, las personas se benefician de los servicios del ecosistema que son los principales contribuyentes al desarrollo de las economías locales y nacionales, se estima que aproximadamente el $65 \%$ de la población mundial viva en zonas urbanas en 2025 (Li; Pan; Wu; Shan, 2017). En la actualidad la expansión urbana es claramente una amenaza para la biodiversidad, cuando se manejan de manera efectiva, los espacios verdes urbanos (parques y jardines) y azules (Pykälä, 2019). Los parques forestales urbanos, que hacen referencia a una colección de árboles dentro o en la periferia de una ciudad con bosques y espacios verdes se manejan cada vez más para propósitos múltiples, entre los cuales el desarrollo recreativo y la conservación ecológica están ganando cada vez más importancia (Zheng; Lan; Chen; Chen; Xu; Chen \& Dong, 2019). Las empresas públicas y privadas utilizan prácticas ecológicas para obtener una ventaja competitiva sostenible, ya que las prácticas ecológicas desempeñan un papel fundamental para establecer una imagen de marca positiva y satisfacer las demandas y expectativas de los clientes (Okumus; Köseoglu; Chan; Hon and Avci, 2019). La rápida expansión de las ciudades que ocupan una gran cantidad de 


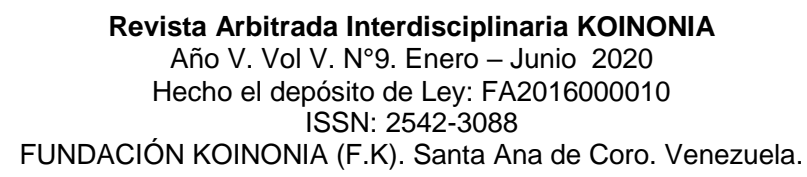

Hilter Farley Figueroa-Saavedra; Arisdorgan Diéguez-Almaguer; Aurora Azucena Ariza-Velasco

tierras agrícolas y paisajes naturales. Sin embargo las ciudades promueven actividades económicas humanas, también traen una serie de problemas naturales, sociales y ambientales como la congestión del tráfico, la contaminación del aire y la crisis de recursos (Liao; Shao; Wang; Tang; Huang and Qiu, 2019). La urbanización altera los sistemas ecológicos, transformando el paisaje biofísico, mientras que la estructura de las ciudades no proporciona muchos servicios eco sistémicos importantes para la biodiversidad y el bienestar humano, los municipios están recurriendo a la restauración ecológica para proporcionar purificación del aire y el agua, reducción de calor y beneficios para la salud y otros ; Los esfuerzos para mejorar la calidad ambiental de los ambientes creados por humanos son inherentemente experimentales (Johnson \& Handel, 2019). Los ecosistemas urbanos representan más del $75 \%$ del consumo mundial de recursos y contribuyen al $80 \%$ de las emisiones de Carbono (ONU-Hábitat, 2016). El parque para recreación infantil, pensado como punto de encuentro social, para niños(as), familias en general y visitantes, además de brindar momentos de distracción y relajación quiere conseguir ayuda económica y bienestar en los habitantes con la implementación de diferentes tipos de materiales obtenidos a base de reciclaje y con el apoyo de energía renovable e inagotable que brinde iluminación a cada uno de sus espacios (Dong; Chen \& Zhang 2017).

El cantón Francisco de Orellana cuenta con un gran potencial turístico, que en su mayor parte está por ser explorado. Entre los atractivos más sobresalientes se encuentran ríos, lagunas, flora, fauna, cascadas, cuevas, paisajes y diferentes culturas indígenas. El desarrollo turístico es reducido todavía, por la falta de capacitación y participación de las comunidades, el limitado acceso a créditos y la escasa promoción desde las instituciones poco coordinadas entre sí.

El estudio se caracteriza para el fortalecimiento de la conciencia medio ambiental de las personas, mediante la implementación de un parque ecológico. Prestará un servicio a los habitantes y turistas que lo visiten, beneficiando a las personas de la localidad que disfrutan de pasar tiempo en compañía de la familia o personas que simplemente 
Revista Arbitrada Interdisciplinaria KOINONIA

Año V. Vol V. N9. Enero - Junio 2020

Hecho el depósito de Ley: FA2016000010

ISSN: 2542-3088

FUNDACIÓN KOINONIA (F.K). Santa Ana de Coro. Venezuela.

Hilter Farley Figueroa-Saavedra; Arisdorgan Diéguez-Almaguer; Aurora Azucena Ariza-Velasco

desean tener un momento de relajación y tranquilidad alejada del estrés de la vida cotidiana.

\section{MATERIALES Y MÉTODOS}

La propuesta de esta investigación se realizó en el cantón Francisco de Orellana, Provincia de Orellana. La temperatura promedio oscila entre 25 a $35{ }^{\circ} \mathrm{C}$, con una pluviosidad anual que va desde 2800 a 4500 mm, el clima es cálido húmedo y una altitud de 200 a 300 m.s.n.m [10].

\section{Diseño de la Investigación.}

Dentro del diseño de la Investigación se hace referencia a la población de estudio, a las variables, a las técnicas e instrumentos de recolección de datos, su validez y confiabilidad.

\section{Población y Muestra}

La población que se utilizó para realizar este trabajo de investigación, fueron todos los estudiantes de las carreras de Turismo y Agronomia de la ESPOCH, Sede Orellana, en un total de 120 personas, y se tomó una muestra de 10 personas para los cálculos estadísticos.

\section{Operacionalización de Variables}

Causa: CREACION DEL PARQUE ECOLOGICO

Efecto: ATRAER TURISMO

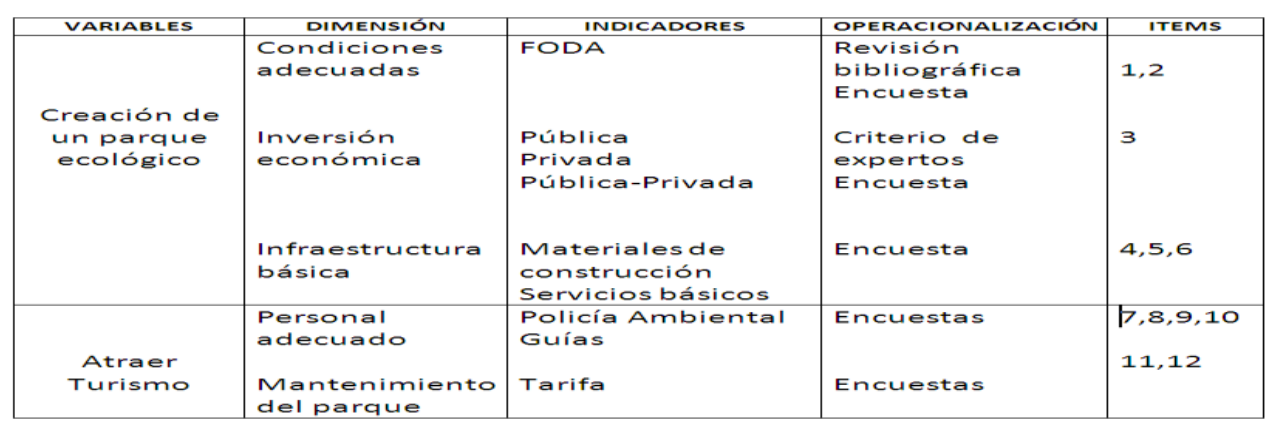




\section{Técnicas e instrumentos de la Recolección de Datos}

A través de la observación se realizó revisiones bibliográficas, conociendo así, que para la creación del Parque Ecológico adscrito al Honorable Consejo Provincial de Orellana, en sesión ordinaria del Consejo llevada a cabo el día 19 de Noviembre del 2018, por decisión unánime de los señores Consejeros adoptan la resolución No. 170-SGHCPO donde se declara en utilidad pública los terrenos de propiedad del señor Daniel Pauker G., en segunda instancia, en sesión ordinaria del 03 de Mayo del 2018 se aprueba la compra del terreno de propiedad mediante la Resolución No 028-SGHCPO, autorizándose a proseguir con los trámites pertinentes los mismos que se encuentran ubicados al margen opuesto del río Payamino, terrenos que serán utilizados para destinarlo a la instalación de un parque ecológico, como parte de la preservación del medio Ambiente y Ecoturismo.

Para la ejecución de esta propuesta, se toma en cuenta la Ley de Gestión ambiental, donde se indica:

Art. 10.- Las instituciones del Estado con competencia ambiental forman parte del Sistema Nacional Descentralizado de Gestión Ambiental y se someterán obligatoriamente a las directrices establecidas por el Consejo Nacional de Desarrollo Sustentable.

Este Sistema constituye el mecanismo de coordinación transectorial, integración y cooperación entre los distintos ámbitos de gestión ambiental y manejo de recursos naturales; subordinado a las disposiciones técnicas de la autoridad ambiental.

La encuesta, aplicada a la muestra determinada y usando como instrumento un cuestionario de 12 preguntas, facilitó la recolección de información

\section{RESULTADOS}

Los resultados de la investigación se resumen en los siguientes: 


\section{Identificación del área a intervenir y sus características.}

Propiedad actual: Daniel Pauker Gutiérrez

Ubicación: Francisco de Orellana

Linderos:

Norte: lote no 2 de José Octavio Vélez - río Payamino

Sur: lote 26 de José Moreira

Este: río Payamino

Oeste: proyecto

Superficie: 59,10 hectáreas

Valor total: 31560,60 dólares

\section{Análisis del coeficiente de confiabilidad}

Para conocer la confiabilidad de las encuestas se calcula aplicando la fórmula de Correlación de Rangos.

\section{Fórmula de Correlación de Rangos}

$$
\begin{aligned}
& r=\frac{n \sum X Y-\sum X \sum Y}{\sqrt{\left.\left[n\left(\sum X^{2}\right)-\left(\sum X\right)^{2}\right] n\left(\sum Y^{2}\right)-\left(\sum Y\right)^{2}\right]}} \\
& r=\frac{120 \sum 96-\sum 97 \sum 103}{\sqrt{\left.\left.120\left(\sum 97\right)-\left(\sum 97\right)^{2}\right] 120\left(\sum 103\right)-\left(\sum 103\right)^{2}\right]}} \\
& r=\frac{11520-9941}{\sqrt{(11640-9409) *(12360-10609)}} \\
& r=\frac{1529}{\sqrt{2231 * 1751}} \\
& r=\frac{1529}{\sqrt{3906481}} \\
& r=\frac{1529}{1976.48}
\end{aligned}
$$


Revista Arbitrada Interdisciplinaria KOINONIA

Año V. Vol V. №9. Enero - Junio 2020

Hecho el depósito de Ley: FA2016000010

ISSN: 2542-3088

FUNDACIÓN KOINONIA (F.K). Santa Ana de Coro. Venezuela.

Hilter Farley Figueroa-Saavedra; Arisdorgan Diéguez-Almaguer; Aurora Azucena Ariza-Velasco $r=0.77$

Una vez obtenido $0.77 \%$ de la correlación según la escala de Guilford (1962), el resultado es de Alta Confiabilidad, representa la realidad de la población a la cual se va a encuestar.

\section{Matriz de resultados de las encuestas}

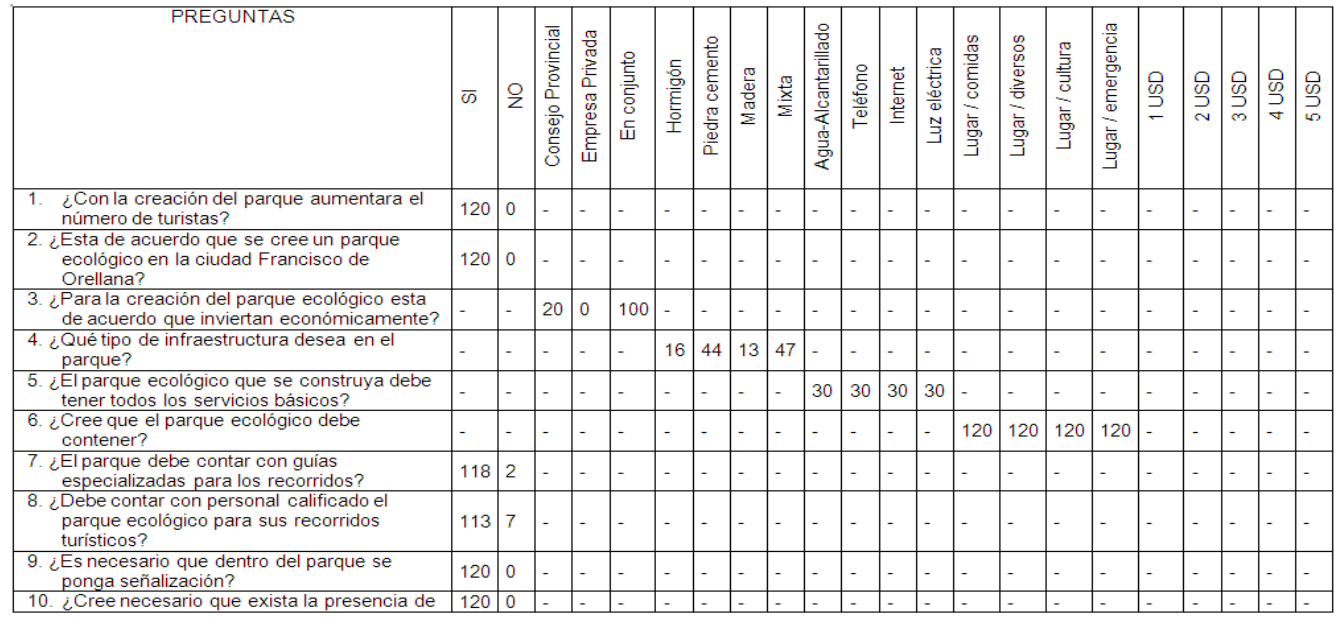

En la matriz de resultados de la encuesta se observa que un promedio del $100 \%$ de la población miran como una opción viable para atraer turistas a la ciudad, esto traerá consigo mayor movimiento en la economía local.

\section{PROPUESTA PARA LA CREACIÓN DEL PARQUE ECOLÓGICO NATURAL "ORELLANA"}

Para realizar la propuesta de creación, se analizan los resultados de factibilidad estructurados en: la caracterización del área, el nivel de confiabilidad y las observaciones obtenidas en las encuestas.

\section{Objetivos de la Propuesta}

General

El proyecto se orienta a dotar a la ciudad de Orellana un espacio público, para la recreación pasiva, la educación y la cultura ambiental. 
Revista Arbitrada Interdisciplinaria KOINONIA

Año V. Vol V. N9. Enero - Junio 2020

Hecho el depósito de Ley: FA2016000010

ISSN: 2542-3088

FUNDACIÓN KOINONIA (F.K). Santa Ana de Coro. Venezuela.

Hilter Farley Figueroa-Saavedra; Arisdorgan Diéguez-Almaguer; Aurora Azucena Ariza-Velasco

\section{Objetivo Ambiental}

Preservar y conservar los elementos y las condiciones del escenario natural existente.

\section{Objetivos Arquitectónicos y Paisajísticos}

- Reforzar las especies vegetativas existentes con un tratamiento adecuado que permita realizar y observar con amplitud cada uno de los elementos de la composición biológica y así integrarla a la arquitectura.

- Implementar una arquitectura paisajística con especies nativas de diferentes alturas, siluetas y densidades, en algunos sectores que lo requieran.

- Plantear una arquitectura orgánica y geométricamente definida para desarrollar un recorrido amable que vigorice el espíritu dentro de un ambiente en concordancia con la naturaleza.

\section{Características del parque}

\section{Condiciones Físicas y Naturales}

El terreno de forma alargada, se ubicará en la periferia de la ciudad.

El área se encuentra servida de dos vías principales que permiten vincularlo a las vías adyacentes al lote.

\section{Características Físicas}

\section{A. Área específica}

Su extensión superficial es de 59.10 hectáreas.

\section{B. Topografía}

El terreno presenta un relieve con características de cañada con pendientes y llanuras prolongadas lo cual permite abrir y cerrar el campo visual.

\section{Vegetación}


Actualmente el predio cuenta con una vegetación espontánea e inducida que se refleja en algunas especies ornamentales que se sembraron con el objeto de mejorar la infraestructura arbórea y consolidar las zonas de fuertes pendientes con barreras vivas como el género de gramíneas denominadas bambú común.

\section{Vientos}

Provienen en dirección norte-sur en intensidad mínima, incluyendo épocas de lluvias.

\section{E. Asoleamiento}

La luz solar es tamizada por el follaje de árboles en un $70 \%$ de la cobertura total del proyecto.

\section{F. Arborización}

Plantaciones de especies inducidas y con características ornamentales como el búcaro, ábaco, guayacán, parasol, melinea.

Taludes naturales conformados por gramíneas como: pasto.

Cerramiento en cerca viva

\section{Distribución del parque}

\section{A. Zona de acceso.}

El acceso se lo hará por vía fluvial, posteriormente se construirá un puente que comunique la cuidad con el parque y dentro del parque se contará con caminos secundarios para los turistas.

Zona de administración - taquilla y baños: En áreas integradas pero separadas estructuralmente, se situarán sobre un terreno plano arborizado y estrecho las dos únicas edificaciones en altura. De esta zona se ramifica y ubica en tres sentidos diferentes los senderos peatonales que colocan al usuario en contacto directo con EL PARQUE.

\section{B. Senderos peatonales y plazoletas}


Revista Arbitrada Interdisciplinaria KOINONIA

Año V. Vol V. N9. Enero - Junio 2020

Hecho el depósito de Ley: FA2016000010

ISSN: 2542-3088

FUNDACIÓN KOINONIA (F.K). Santa Ana de Coro. Venezuela.

Hilter Farley Figueroa-Saavedra; Arisdorgan Diéguez-Almaguer; Aurora Azucena Ariza-Velasco

Los senderos y plazoletas tendrán las siguientes funciones:

\section{Pasiva}

Contemplación de la belleza del paisaje

Lectura y pintura

Descanso

Meditación y encuentro con la naturaleza, con los demás seres humanos y consigo mismo.

\section{Activa}

Caminar

Trotar

Eventos artísticos y culturales

Contará con los siguientes elementos arquitectónicos:

Sendero principal

Sendero perimetral

Plazoletas

\section{Zona aventuras y escalera de esfuerzo}

Este sitio cuenta con un sendero ecológico que recorre todo el parque. Es ideal para hacer una caminata ecológica en un ambiente lleno de árboles como búcaros, abarcos, guayacanes, parasoles, melíneas, oitís y bambúes. Además, cuenta con una zona de aventura y escalera de esfuerzo, zona de juegos infantiles y un kiosko

\section{Zona de juegos infantiles:}

Kiosco

Mobiliario Urbano

Paisajismo 
Revista Arbitrada Interdisciplinaria KOINONIA

Año V. Vol V. №9. Enero - Junio 2020

Hecho el depósito de Ley: FA2016000010

ISSN: 2542-3088

FUNDACIÓN KOINONIA (F.K). Santa Ana de Coro. Venezuela.

Hilter Farley Figueroa-Saavedra; Arisdorgan Diéguez-Almaguer; Aurora Azucena Ariza-Velasco

\section{Promoción y Publicidad}

Se establecerá una promoción local, regional y nacional del Parque Ecológico Natural Orellana, para informar sobre los atractivos que contará el lugar.

Se utilizará los medios de comunicación como: radio, televisión, boletines de prensa, elaboración de trípticos, afiches, etc.

Se estima que cada semana el parque reciba visitantes de diferentes edades y estratos sociales de la ciudad de Orellana y la provincia, por sus diversos escenarios, además se espera la visita al parque de las personas del extranjero.

El Parque Ecológico Natural Orellana establecerá convenios con el Ministerio del Ambiente a través de la Oficina Técnica de Orellana con la finalidad de que las especies animales y plantas que son decomisadas, las integren nuevamente a su hábitat natural. En base a estos convenios, se contará con la ayuda de Fundaciones dedicadas a la conservación de la biodiversidad como Fundación Natura y Jatún Sacha, para que colaboren con personal técnico, biólogos, forestales y botánicos para que se realicen investigaciones con la flora y fauna.Con la Policía Nacional, con su grupo especializado en turismo para que colaboren con la seguridad.

\section{Modalidad de administración}

La administración ha sido concebida a la entrega de servicios de alta calidad a todos sus visitantes. Se propone el siguiente organigrama:

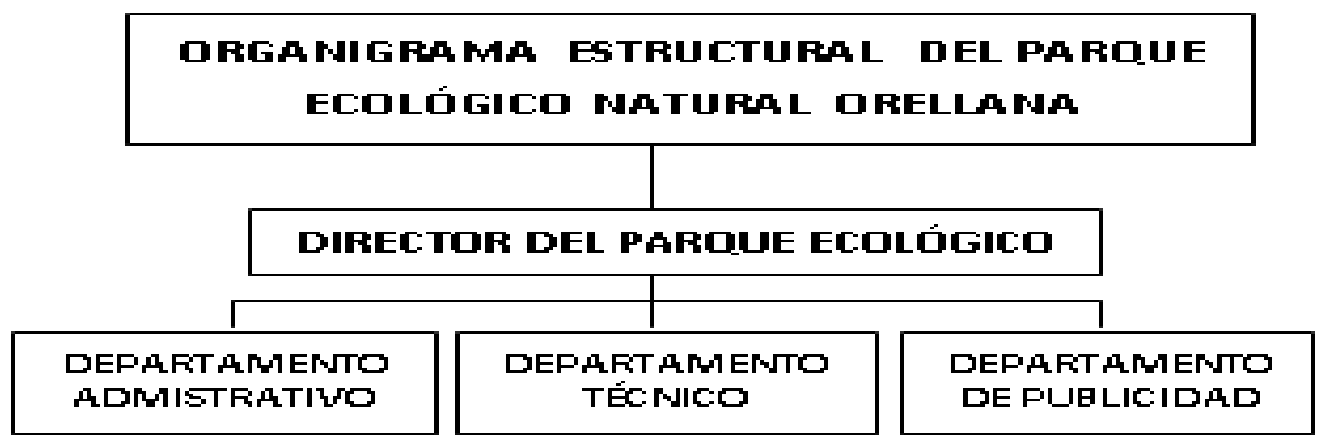

Cada uno de los componentes del Organigrama se regirá a las funciones técnicas. 
Revista Arbitrada Interdisciplinaria KOINONIA

Año V. Vol V. N9. Enero - Junio 2020

Hecho el depósito de Ley: FA2016000010

ISSN: 2542-3088

FUNDACIÓN KOINONIA (F.K). Santa Ana de Coro. Venezuela.

Hilter Farley Figueroa-Saavedra; Arisdorgan Diéguez-Almaguer; Aurora Azucena Ariza-Velasco

\section{Factibilidad de la Propuesta}

Económica: Se cuenta directamente con el visto bueno del Consejo Provincial de Orellana y además se está ejecutando acciones para contar con el apoyo de diferentes ONG's.

Legal: Se orienta directamente a la Ley del Régimen Provincial, y de la Ley Forestal y Conservación de Áreas Naturales y Silvestres.

Humano: Se cuenta con el personal tanto interno como externo del Consejo Provincial de Orellana.

\section{CONCLUSIONES}

La investigación para la creación del Parque Ecológico sigue un proceso metodológico que empieza con la identificación de la población y muestra, análisis de las variables y el coeficiente de confiabilidad para su ejecución.

De acuerdo a los resultados de la investigación, el proyecto es factible por disponer un área adjudicada de 59,10 hectáreas, estratégicamente ubicada y con características excepcionales que ha permitido realizar una distribución de instalaciones e infraestructura de acuerdo a su contexto y objetivos del proyecto

La propuesta de creación del Parque Ecológico Natural "Orellana" es una alternativa excelente, estructurada de acuerdo a la Ley del Régimen Provincial, y de la Ley Forestal y Conservación de Áreas Naturales y Silvestres que ejecutará el Honorable Consejo Provincial para incrementar el turismo en la provincia de Orellana, en el que se dará un uso adecuado de los recursos naturales y una disposición de talento humano para su administración. 


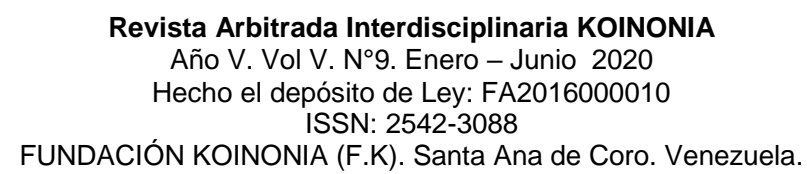

Hilter Farley Figueroa-Saavedra; Arisdorgan Diéguez-Almaguer; Aurora Azucena Ariza-Velasco

\section{Recomendaciones}

Se recomienda Tomar en cuenta el coeficiente de confiabilidad para determinar las actividades de ejecución y el alcance de metas planteadas.

Se recomienda utilizar técnicamente la amplitud de la superficie y la diversidad de recursos para cumplir con el objetivo de proteger y aprovechar las áreas naturales.

Se recomienda ejecutar la propuesta de creación del Parque Ecológico Natural de "Orellana" para incrementar el turismo y mejorar a través de ello, el nivel de vida de la población local.

\section{FINANCIAMIENTO}

No monetario.

\section{AGRADECIMIENTOS}

Al Honorable Consejo Provincial de Orellana por el apoyo en el desarrollo de este proyecto investigativo.

\section{REFERENCIAS CONSULTADAS}

Monz, C., D'Antonio, A., Lawson, S., Barber, J. and Newman, P. (2016). The ecological implications of visitor transportation in parks and protected areas: Examples from research in US National Parks. [Las implicaciones ecológicas del transporte de visitantes en parques y áreas protegidas: ejemplos de investigaciones en parques nacionales de EE. UU]. Journal of Transport Geography, 51, pp.27-35.

Li, F., Pan, B., Wu, Y. and Shan, L. (2017). Application of game model for stakeholder management in construction of ecological corridors: A case study on Yangtze River Basin in China. [Aplicación del modelo de juego para la gestión de los interesados en la construcción de corredores ecológicos: un estudio de caso sobre la cuenca del río Yangtze en China]. Habitat International, 63, pp.113-121.

Pykälä, J. (2019). Habitat loss and deterioration explain the disappearance of populations of threatened vascular plants, bryophytes and lichens in a hemiboreal landscape. [La pérdida y el deterioro del hábitat explican la desaparición de poblaciones de plantas vasculares, briófitos y líquenes amenazados en un paisaje hemiboreal.]. Global Ecology and Conservation, 18, p.e00610. 
Hilter Farley Figueroa-Saavedra; Arisdorgan Diéguez-Almaguer; Aurora Azucena Ariza-Velasco

Zheng, Y., Lan, S., Chen, W. Y., Chen, X., Xu, X., Chen, Y., \& Dong, J. (2019). Visual sensitivity versus ecological sensitivity: An application of GIS in urban forest park planning. . [Sensibilidad visual versus sensibilidad ecológica: una aplicación de SIG en la planificación de parques forestales urbanos]. Urban Forestry \& Urban Greening, 41, 139-149. https://doi.org/https://doi.org/10.1016/j.ufug.2019.03.010

Okumus, F., Köseoglu, M., Chan, E., Hon, A. and Avci, U. (2019). How do hotel employees' environmental attitudes and intentions to implement green practices relate to their ecological behavior?. [¿Cómo se relacionan las actitudes e intenciones ambientales de los empleados del hotel para implementar prácticas ecológicas con su comportamiento ecológico?]. Journal of Hospitality and Tourism Management, 39, pp.193-200.

Liao, J., Shao, G., Wang, C., Tang, L., Huang, Q. and Qiu, Q. (2019). Urban sprawl scenario simulations based on cellular automata and ordered weighted averaging ecological constraints. [Simulaciones de escenarios de expansión urbana basados en autómatas celulares y restricciones ecológicas promedio ponderadas ordenadas]. Ecological Indicators, 107, p.105572.

Johnson, L. R., \& Handel, S. N. (2019). Management intensity steers the long-term fate of ecological restoration in urban woodlands. [La intensidad del manejo dirige el destino a largo plazo de la restauración ecológica en los bosques urbanos]. Urban Forestry \& Urban Greening, 41, 85-92. https://doi.org/https://doi.org/10.1016/j.ufug.2019.02.008

ONU-Hábitat, (2016). Urbanización y desarrollo deONU-Hábitat: futuros emergentes. Informe de ciudades del mundo 2016. [Urbanization and development of UNHabitat: emerging futures. World Cities Report 2016]. Programa de las Naciones Unidas para los Asentamientos Humanos ( 2016 ). http://www.unhabitat.org

Dong, N., Chen, J., \& Zhang, S. (2017). Safety Research of Children's Recreational Space in Shanghai Urban Parks. [Investigación de seguridad del espacio recreativo para niños en los parques urbanos de Shanghai]. Procedia Engineering, 198 , https://doi.org/https://doi.org/10.1016/j.proeng.2017.07.115

612-621.

GADPO, 2015: "Plan de Desarrollo y Ordenamiento Territorial de la Provincia de Orellana 2015-2019. [Plan of Development and Territorial Planning in Orellana Province, 2015-2019].

BANCO BILBAO-VIZCAYA. (1993). Espacios Naturales Protegidos. [Natural Protected Areas].El Campo no 128 
Revista Arbitrada Interdisciplinaria KOINONIA

Año V. Vol V. N9. Enero - Junio 2020

Hecho el depósito de Ley: FA2016000010

ISSN: 2542-3088

FUNDACIÓN KOINONIA (F.K). Santa Ana de Coro. Venezuela.

Hilter Farley Figueroa-Saavedra; Arisdorgan Diéguez-Almaguer; Aurora Azucena Ariza-Velasco

Benayas, J Y Heras, F.(1994). Ideas para interpretar y mejorar nuestros paisajes. En (coord.) Viviendo el paisaje. [Ideas to interpret and improve our landscapes. In (coord.) Living the landscape]. Fundación Natwest. Madrid.

Busot, A. (1991). Investigación Educacional. Venezuela. [Educational Research, Venezuela]. Universidad de Zulia.

Clapham, V. (1987). Hacer para comprender. [Make people understand]. Fichas de experimentos para la conservación. ADENA-WWF.

Diccionario Encarta 2004. [Encarta Dictionary].

Escuela Superior Politécnica Ecológica Amazónica, (2003) Estatuto Orgánico. [Organic Statute].

ICONA (1994). Parques para la vida. Plan de acción para las áreas protegidas de Europa. [Parks for life. Action plan for Europe's protected areas]. UICN.

Marcano, J. Fecha desconocida. ¿Qué es la Ecología? Página de Educación Ambiental: Elementos de Ecología. [What is ecology? Environmental Education Page: Elements of Ecology]. (http://jmarcano.vr9.com/nociones/quees.html), revisada el 13 de agosto de 2001.

Siguero, P. L. (1991). Consejos y recomendaciones para reforestar con especies autóctonas. [Tips and recommendations for reforestation with native species]. Quercus no 62 y 63. 